\title{
Recognising and managing antidepressant discontinuation symptoms
}

\author{
Peter M. Haddad \& Ian M. Anderson
}

\begin{abstract}
Antidepressant discontinuation symptoms occur with all classes of antidepressant. A well-described discontinuation syndrome occurs with the selective serotonin reuptake inhibitors, common symptoms including dizziness, headache, nausea and lethargy. Rare antidepressant discontinuation syndromes include extrapyramidal syndromes and mania/hypomania. All these syndromes, even isolated discontinuation symptoms, share three common features that facilitate diagnosis; abrupt onset within days of stopping the antidepressant, a short duration when untreated and rapid resolution when the antidepressant is reinstated. Clinicians need to be familiar with strategies for the prevention and management of such symptoms. Preventive strategies include warning patients about the possibility of discontinuation symptoms, encouraging good antidepressant adherence and tapering antidepressants at the end of treatment. Most symptoms are mild and short-lived. Consequently symptoms that follow planned termination of an antidepressant can often be managed by providing an explanation and reassurance. More severe symptoms should be treated symptomatically or the antidepressant restarted, in which case symptoms usually resolve within $24 \mathrm{~h}$. More cautious tapering can then follow.
\end{abstract}

Antidepressant discontinuation (withdrawal) symptoms were first reported in association with imipramine (Mann \& MacPherson, 1959; Andersen \& Kristiansen, 1959), the first tricyclic antidepressant (TCA), shortly after it entered clinical use. These symptoms occur with all classes of antidepressant, including the TCAs, monoamine oxidase inhibitors (MAOIs), selective serotonin reuptake inhibitors (SSRIs), serotonin and noradrenaline reuptake inhibitors (SNRIs) and miscellaneous others such as mirtazapine, a noradrenergic and specific serotonergic antidepressant (NaSSA). A PubMed review conducted when preparing this article identified reports of discontinuation symptoms with 25 antidepressants (Box 1). In recent years the phenomenon has attracted increasing interest, in both the scientific literature and in the lay media (BBC Panorama, 2002, 2003). This article provides an overview of the clinical features of antidepressant discontinuation symptoms, with the emphasis on their recognition, prevention and management. Discontinuation symptoms can occur whenever antidepressants are used, i.e. they are not dependent on the presence of any underlying psychiatric disorder. The pharmacokinetics and dynamics of antidepressants, in particular their halflife, are important determinants of discontinuation symptoms, as are individual patient characteristics, but their discussion is beyond the scope of this article (Schatzberg et al, 1997; Haddad, 1998; Bogetto et al, 2002).

\section{The question of addiction}

The terms 'antidepressant discontinuation symptom' and 'antidepressant withdrawal symptom' are used interchangeably in the literature. 'Discontinuation' is preferred by some authorities, as it does not imply that antidepressants are addictive or cause a dependence syndrome, whereas the term 'withdrawal' may imply this. Both terms are likely to remain in use and it is more important to be clear about what they refer to rather than which is preferable.

The occurrence of withdrawal symptoms does not in itself indicate that a drug causes dependence as defined in ICD-10 (World Health Organization, 1992)

Peter Haddad is a consultant psychiatrist with Bolton, Salford and Trafford Mental Health NHS Trust (Cromwell House, Cromwell Road, Eccles, Salford, Manchester M30 0GT, UK. Email: peter.haddad@bstmht.nhs.uk) and an honorary senior lecturer at the University of Manchester. His clinical and research interests include the pharmacological treatment of affective disorders and schizophrenia and the safety and adverse effects of psychotropic medication. He was a member of the Guideline Development Group for the National Institute for Health and Clinical Excellence's guidelines on bipolar disorder (2006). Ian Anderson is Senior Lecturer in Psychiatry at the University of Manchester and an honorary consultant psychiatrist with Manchester Mental Health and Social Care Trust, where he is Director of the Specialist Service for Affective Disorders. His clinical and research interests include the neurobiology and pharmacological treatment of bipolar and unipolar affective disorders. He has been involved in the British Association for Psychopharmacology's evidence-based guidelines for depression, anxiety disorders and bipolar disorder. 


\section{Box 1 Antidepressants reported to cause dis- continuation symptoms}

Tricyclic and related compounds

- Amineptine

- Amitriptyline

- Amoxapine

- Clomipramine

- Desipramine

- Doxepin

- Imipramine

- Nortriptyline

- Protriptyline

- Trazodone

Monoamine oxidase inhibitors

- Isocarboxazid

- Moclobemide

- Phenelzine

- Tranylcypromine

Selective serotonin reuptake inhibitors

- Citalopram

- Escitalopram

- Fluoxetine

- Fluvoxamine

- Paroxetine

- Sertraline

Serotonin and noradrenaline reuptake inhibitors

- Duloxetine

- Milnacipran

- Venlafaxine

Miscellaneous antidepressants

- Mirtazapine (noradrenergic and specific serotonergic antidepressant, NaSSA)

- Nefazodone

(Based on the authors' literature review)

and DSM-IV (American Psychiatric Association, 1994). An extensive report published by the Committee on Safety of Medicines concluded that

'there is no clear evidence that the SSRIs and related antidepressants have a significant dependence liability or show development of a dependence syndrome according to internationally accepted criteria (either DSM-IV or ICD-10)' (Committee on Safety of Medicines, 2004: p. 3).

This is consistent with earlier publications examining this area (Haddad, 1999; Haddad \& Anderson, 1999; Tyrer, 1999), despite the arguments to the contrary put forward by some critics (Medawar, 1997).

When considering whether antidepressants are addictive or dependence-forming it is helpful to step back and consider why a label or diagnosis is important to patients. For patients the main advantage of a diagnosis is that it can help predict the treatment and future course of a disorder, i.e. it allows a prognosis to be given. Craving and relapse are common features of dependence on alcohol, opiates and stimulants and both can occur after long periods of abstinence. At a practical level it is the relapsing nature of dependence that makes it so problematic for patients and clinicians. In contrast, managing withdrawal symptoms in those with substance dependence is not the key problem as such symptoms are time-limited. There is no evidence that patients crave antidepressants once they have stopped them or feel compelled to return to taking antidepressants once any discontinuation symptoms, if they occur, have ceased. This difference in prognosis, or long-term course, to that of the syndrome of dependence seen with alcohol, opiates and stimulants seems easily understandable by patients. For a full discussion about whether antidepressants cause dependence see Haddad (2005), in which it is concluded that antidepressants have no significant liability to cause dependence as defined in ICD-10 and DSM-IV.

\section{Clinical relevance}

Antidepressant discontinuation symptoms are important as they can cause morbidity, affect adherence to antidepressant treatment, prevent antidepressants being stopped and can be misdiagnosed, leading to inappropriate treatment. These adverse outcomes are discussed further in this section.

In most patients, discontinuation symptoms are self-limiting, of short duration and mild, but in a minority of cases they can be severe, last several weeks and cause significant morbidity. For example, case reports of SSRI discontinuation reactions have described ataxia leading to falls (Einbinder, 1995), fatigue causing difficulty walking (Haddad et al, 2001) and electric-shock-like sensations impairing walking and driving (Frost \& Lal, 1995). Occasionally discontinuation symptoms lead to urgent consultations and attendance at accident and emergency departments (Pacheco et al, 1996; Haddad et al, 2001).

Poor adherence is common and some patients miss consecutive doses of antidepressants for several days (Demyttenaere et al, 2001). Such breaks may precipitate discontinuation symptoms. Some patients find this beneficial as the symptoms act as a reminder to take medication. However, the experience may cause others to stop their antidepressant permanently, particularly if they worry that the discontinuation symptoms indicate that they are becoming 'addicts'. Thus discontinuation symptoms can result from, and also cause, poor adherence. 
Severe discontinuation symptoms can hinder or even prevent patients stopping antidepressant treatment: symptoms can be so unpleasant that the patient has to restart the antidepressant to stop the symptoms. How often this occurs, and why some patients experience severe problems when most do not, is unknown and warrants further investigation (Schatzberg et al, 2006). It has been argued that this phenomenon demonstrates that antidepressants are addictive (Medawar, 1997) but, as discussed above, withdrawal or discontinuation symptoms on their own are insufficient to define dependence in the clinically accepted sense of the word.

An important clinical aspect of antidepressant discontinuation symptoms is the potential for their misdiagnosis, as either a physical or a psychiatric disorder, leading to the offer of inappropriate management for the 'incorrect' diagnosis. In particular, discontinuation symptoms may be diagnosed as a relapse or recurrence of the underlying affective illness for which the antidepressant was originally prescribed, although other scenarios exist (Box 2). It is not known how often misdiagnosis occurs. In our experience it usually results from clinicians' unfamiliarity with antidepressant discontinuation syndromes rather than the symptoms being difficult to diagnose.

\section{Core clinical features}

\section{Nature of symptoms}

Antidepressant discontinuation symptoms are diverse and differ between antidepressant classes. A review of published case reports of SSRI discontinuation reactions identified over 50 different symptoms (Haddad, 1998). Discontinuation reactions occur on a spectrum in terms of both the number

\section{Box 2 Examples of the misdiagnosis of antidepressant discontinuation symptoms}

\section{Misdiagnosis as adverse effect of new medication}

Discontinuation symptoms that follow antidepressant switching may be incorrectly diagnosed as sideeffects of the new antidepressant (Haddad \& Qureshi, 2000), which may be stopped on the assumption that the patient cannot tolerate it. This scenario usually occurs when switching across antidepressant classes; discontinuation symptoms are uncommon when switching between antidepressants with similar mechanisms of action.

\section{Misdiagnosis as recurrence of the underlying psychiatric illness}

Discontinuation symptoms that follow recovery from a depressive illness and termination of antidepressant treatment may be misdiagnosed as a recurrence of depression, i.e. a further depressive episode. This may lead to unnecessary reinstatement of the antidepressant and a more negative prognosis, with significant social implications. The same effect can occur when antidepressants are used to treat other psychiatric disorders, for example discontinuation symptoms may be misdiagnosed as a recurrence of generalised anxiety disorder or panic disorder.

\section{Misdiagnosis as failure to respond to treatment}

Discontinuation symptoms due to covert non-adherence to acute antidepressant treatment may be mistaken as worsening of the underlying illness (depression or other psychiatric disorders) and lead the doctor to incorrectly conclude that treatment is ineffective. As a result the antidepressant dose may be increased, an augmentation strategy adopted or an unnecessary switch made to another antidepressant.

\section{Misdiagnosis of discontinuation mania and hypomania}

Manic and hypomanic symptoms occur as rare antidepressant discontinuation symptoms. If a patient with unipolar depression develops such symptoms, but it is not recognised that they are discontinuation reactions, an erroneous diagnosis of bipolar I or bipolar II disorder may be made and the patient inappropriately started on long-term treatment with a mood stabiliser.

\section{Misdiagnosis as physical disorder}

Many discontinuation symptoms are physical. Failure to diagnose them may lead to unnecessary referrals and investigations in an attempt to identify a 'physical' problem. Examples include a case in which neurological symptoms due to paroxetine discontinuation led to a neurology referral, a computerised tomographic brain scan and electroencephalograph (Haddad et al, 2001) and a case in which dizziness due to fluoxetine discontinuation led to an ear, nose and throat referral and magnetic resonance imaging of the head (Einbinder, 1995). 
and severity of symptoms, ranging from an isolated symptom to a cluster and from mild to severely disabling. This raises the issue of a diagnostic threshold. However, there is no accepted definition of an antidepressant discontinuation syndrome, although operational criteria have been proposed (Haddad, 1998; Black et al, 2000). The occurrence of different symptom clusters, or discontinuation syndromes, adds a further level of complexity. A socalled 'primary' ('general') discontinuation syndrome is the most common syndrome encountered with the SSRIs and SNRIs. Extrapyramidal syndromes and mania/hypomania can occur as rare discontinuation syndromes with various classes of antidepressant. All these syndromes, and even isolated discontinuation symptoms, appear to share several common features: time of onset of symptoms relative to stopping the antidepressant, duration of symptoms when untreated and response to restarting the antidepressant. These general characteristics are discussed further in this section, and specific syndromes are discussed in the subsequent section.

\section{Time of onset}

Discontinuation symptoms usually appear within a few days of stopping an antidepressant or, less commonly, reducing the dose. Onset of symptoms after more than 1 week is unusual. In a naturalistic study of 97 patients the mean time of onset of discontinuation symptoms was 2 days after stopping an SSRI (Bogetto et al, 2002). In a series of 160 adverse drug reaction reports of paroxetine discontinuation reactions the median interval between stopping paroxetine and symptom onset was 2.1 days (Price et al, 1996). Within this data-set symptoms occurred within 4 days in $86 \%$ and within 1 week in $93 \%$.

\section{Duration}

Most antidepressant discontinuation reactions are of short duration, resolving spontaneously between 1 day and 3 weeks after onset. Bogetto et al (2002) reported that the mean duration of SSRI discontinuation symptoms was 5 days. In 71 untreated paroxetine discontinuation reactions reported by doctors as adverse drug reactions (Price et al, 1996), and presumably representing the severer end of the spectrum, the median duration was 8 days (range $1-52$ days).

\section{Effect of restarting medication}

Clinical experience is that discontinuation symptoms usually resolve fully within $24 \mathrm{~h}$ if the original antidepressant is recommenced. Several studies have demonstrated the resolution of symptoms at assessment 1 week after reinstatement of the original antidepressant (Rosenbaum et al, 1998) or a pharmacologically similar one (Tint et al, 2007).

\section{Specific syndromes \\ Primary SSRI discontinuation syndrome}

This is the most common discontinuation syndrome seen with the SSRIs. The term 'primary' (or 'general') is used to differentiate it from rare SSRI discontinuation syndromes such as extrapyramidal syndromes and mania. The physical and psychological symptoms of primary SSRI discontinuation syndrome can be divided into six groups (Fig. 1). The most common symptoms are dizziness, nausea, lethargy and headache (Haddad, 1998). Some patients experience sensory symptoms (e.g. sensations resembling electric shocks) or symptoms of disequilibrium (e.g. dizziness) in brief bursts when they move their head or eyes. Such symptoms are highly characteristic of primary discontinuation syndrome. The syndrome was initially reported in case reports and adverse drug reaction reports (Haddad, 1998) but its features have been confirmed in several double-blind studies in which SSRI treatment is briefly interrupted with placebo (Rosenbaum et al, 1998; Michelson et al, 2000; Judge et al, 2002). A similar discontinuation syndrome occurs with venlafaxine (Fava et al, 1997) and duloxetine (Perahia et al, 2005).

\section{Primary TCA discontinuation syndrome}

This shares four of the six SSRI symptom groups (Fig. 1). The remaining two groups, sensory abnormalities and problems with equilibrium, seem to be less common with TCAs and can be regarded as SSRI specific. It is unclear whether primary SSRI and TCA discontinuation syndromes would be better regarded as several sub-syndromes.

\section{MAOI discontinuation syndrome}

Reactions to MAOI discontinuation, particularly those reported with tranylcypromine, tend to be more severe than with other antidepressants. The clinical picture may include: (i) a worsening of depressive symptoms, exceeding the severity of the state that originally led to treatment (Halle \& Dilsaver, 1993); (ii) an acute confusional state with disorientation, paranoid delusions and hallucinations (Liskin et al, 1984; Roth, 1985; Halle \& Dilsaver, 1993); and (iii) anxiety symptoms, including hyperacusis and depersonalisation (Tyrer, 1984). 


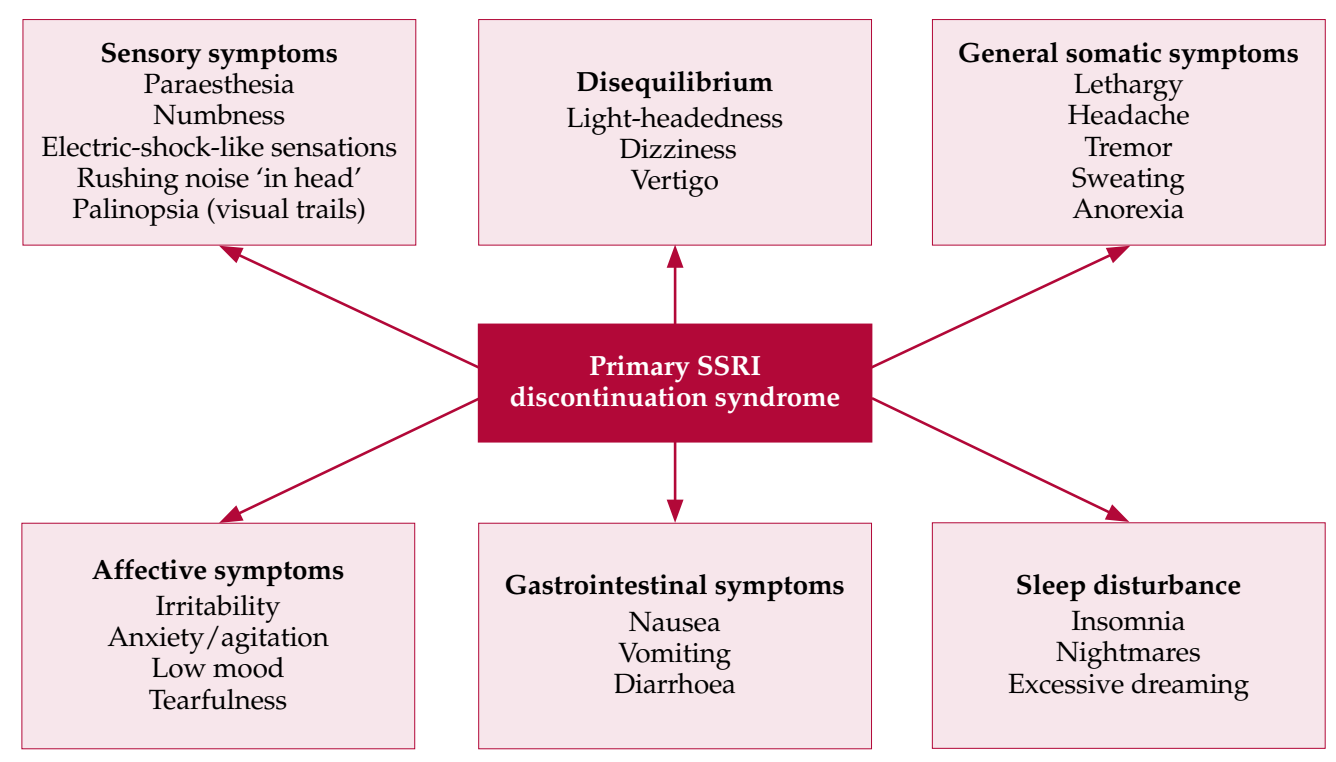

Fig. 1 Key symptom groups of primary SSRI discontinuation syndrome. Common or characteristic symptoms are listed, but many others are reported. Patients vary in the number and combination of symptoms they manifest.

\section{Rare syndromes}

Case reports have described a variety of reactions to discontinuation of antidepressants, including extrapyramidal syndromes and mania/hypomania. The incidence of these syndromes is unknown, but the fact that they have not been observed in clinical studies suggests that they are uncommon. Sudden onset of mania/hypomania has been reported with termination of TCAs (e.g. Mirin et al, 1981), SSRIs (e.g. Szabadi, 1992; Bloch et al, 1995), MAOIs (e.g. Rothschild, 1985), venlafaxine (Goldstein et al, 1999) and mirtazapine (MacCall \& Callender, 1999). The phenomenon has been reported in patients with unipolar depression and bipolar disorder. Parkinsonian symptoms have been reported following missed doses of desipramine (Dilsaver et $a l, 1983 a$ ), dystonia on stopping fluoxetine (Stoukides \& Stoukides, 1991), and akathisia on stopping venlafaxine (Wolfe, 1997), fluvoxamine (Hirose, 2001) and imipramine (Sathananthan \& Gershon, 1973). Various other discontinuation symptoms have occasionally been reported, but it is difficult to be sure that the relationship with drug termination is causal rather than a spurious association.

\section{Incidence}

Several factors influence the incidence of discontinuation symptoms. Symptoms are more common when higher antidepressant doses are stopped (Committee on Safety of Medicines, 2004; Perahia et al, 2005) and when the duration of treatment has been longer (e.g. Kramer et al, 1961). However, a plateau in incidence appears to be reached fairly early on in treatment, with duration beyond this resulting in no further increase (Perahia et al, 2005; Baldwin et al, 2007). For example in a review of duloxetine studies, extended treatment with the drug beyond 8-9 weeks did not appear to be associated with an increased incidence or severity of symptoms (Perahia et al, 2005). Intuitively it makes sense that symptoms would be more likely if the antidepressant were stopped abruptly rather than tapered down, although such an effect has not been demonstrated in clinical studies. The methodology by which discontinuation symptoms are defined and assessed will also influence incidence rates.

Discontinuation symptoms are a common occurrence with many antidepressants. Fava et al (1997) reported that during the 3 days following stoppage of venlafaxine and placebo under double-blind conditions, seven $(78 \%)$ of nine participants treated with venlafaxine and two $(22 \%)$ of nine placebotreated individuals reported the emergence of adverse events, a statistically significant difference. Tint et al (2007) reported that 13 of 28 patients (46\%) fulfilled criteria for a discontinuation syndrome (defined as the onset of three or more new symptoms on a checklist) when assessed 5-7 days after stopping an SSRI or venlafaxine.

Antidepressants differ in their propensity to cause discontinuation symptoms. For example in a 6-week double-blind study that compared milnacipran with paroxetine both drugs were equally effective in treating depression, but after treatment 
discontinuation, paroxetine was associated with significantly more discontinuation symptoms (Vandel et al, 2004). Among the SSRIs several prospective studies have show that paroxetine is associated with the highest incidence of discontinuation symptoms and fluoxetine the lowest (Rosenbaum et al, 1998; Michelson et al, 2000; Bogetto et al, 2002; Judge et al, 2002; Tint et al, 2007).

\section{Differential diagnosis}

The three key features that suggest the diagnosis of an antidepressant discontinuation syndrome are: (i) the abrupt onset of (ii) characteristic symptoms (iii) within a few days of an antidepressant being stopped or reduced in dose. A discontinuation syndrome that occurs when an antidepressant is stopped following recovery from a depressive illness must be distinguished from a recurrence (i.e. a new episode) of depression. If there are doubts over diagnosis, and symptoms are not severe, then the clinician and patient can monitor the course of the symptoms and reserve a definitive diagnosis to a later date. If adopting this approach the clinician should give a full explanation to the patient. A sudden onset of symptoms with spontaneous resolution within about 10 days is the norm with discontinuation reactions (Price et al, 1996; Bogettto et al, 2002). In contrast, one would expect symptoms of a depressive illness to start more gradually, worsen with time and be more persistent; a 2-week duration of symptoms is mandatory for a diagnosis of a major depressive episode in DSM-IV (American Psychiatric Association, 1994).

\section{Mania}

Mania that occurs after a patient with unipolar depression has stopped taking an antidepressant may be a discontinuation reaction or it may be independent of antidepressant stoppage and an indicator of bipolar I disorder. The onset of symptoms within days of antidepressant stoppage would strongly suggest a discontinuation syndrome. As the manic symptoms are identical irrespective of the aetiology, differentiation is not as clear as when differentiating the primary SSRI or TCA discontinuation syndromes from a recurrence of depression. In the latter case characteristic symptoms such as dizziness and paraesthesia, which are uncommon in a depressive illness, can facilitate the diagnosis of a discontinuation syndrome. If the treatment adopted for presumed 'discontinuation mania' is antidepressant reinstatement then it is advisable to monitor the patient closely, ideally as an in-patient, because if the diagnosis is incorrect and the patient has a bipolar disorder then the antidepressant may exacerbate the manic symptoms. If there is doubt about the aetiology it seems best to treat the mania symptomatically using an antipsychotic rather than to restart the antidepressant.

\section{Non-adherence}

Discontinuation symptoms are not confined to the cessation of an antidepressant on a doctor's advice. Non-adherence to antidepressant treatment is common and often covert unless inquired about. Consequently a discontinuation syndrome should also be considered when unexpected physical or psychological symptoms arise in a patient prescribed an antidepressant. In such cases it is important to ask the patient, in a non-critical manner, whether they are taking the medication as prescribed and, if not, about the relationship between missed doses, the onset and continuation of symptoms. When physical symptoms predominate, clinical judgment will determine when a physical examination and investigations, particularly blood tests, are needed to exclude physical disorders.

\section{Prevention}

\section{Tapering after successful treatment}

Tapering antidepressants at the end of treatment, rather than abrupt stoppage, is recommended as standard practice by several authorities and treatment guidelines (Drug and Therapeutics Bulletin, 1999; Anderson et al, 2000; British Medical Association \& Royal Pharmaceutical Society of Great Britain, 2007) and in the summary of product characteristics of many antidepressants. Recommendations on taper length vary. For example the British National Formulary (BNF; British Medical Association \& Royal Pharmaceutical Society of Great Britain, 2007: p. 200) recommends that antidepressants administered for 8 weeks or more should, wherever possible, be reduced over a 4-week period. Other authorities recommend more cautious tapers. However, there are no controlled data to recommend the effectiveness of tapering, the length of time over which it should occur or the minimum dose that one should taper to before cessation (Tint et al, 2007).

The only randomised published study to have assessed the effect of duration of tapering on discontinuation symptoms is Tint et al (2007). This open study recruited patients with major depressive disorder in whom the treating clinician wanted to switch the existing antidepressant, either an SSRI or venlafaxine, owing to lack of response. Participants completed standard scales assessing discontinuation symptoms and depressive symptoms at baseline 
(on their original antidepressant) and after a 3-day or 14-day taper followed by a washout period (no antidepressant prescribed) of 5-7 days. There was a significant increase in both depressive symptoms (including suicidal ideation) and discontinuation symptoms between baseline and assessment at the end of the drug-free period, with a 'discontinuation syndrome' (> 3 new symptoms) occurring in $46 \%$ of patients. However, taper duration had no significant effect on the increase in either discontinuation symptoms or depressive symptoms or the incidence of a discontinuation syndrome. Owing to the design of this study it is impossible to know whether abruptly stopping the antidepressant would have led to more discontinuation symptoms than the 3-day taper. The results also imply that if tapering SSRIs and venlafaxine is beneficial in reducing symptoms, and intuitively one would expect it to be, then its duration needs to be in excess of 14 days for most patients.

The only evidence that tapering antidepressants is of benefit comes from several case reports that describe discontinuation symptoms being suppressed by reintroduction of the antidepressant, with a subsequent taper preventing their re-emergence (e.g. Dominguez \& Goodnick, 1995; Benazzi, 1996). In practice it seems that individuals vary greatly in their propensity to experience discontinuation symptoms and the duration of taper that is required to prevent such symptoms.

When antidepressants are stopped at the end of a successful treatment period, without any intention to switch, then there is no time pressure to limit the duration of the taper. Some of the factors that will influence the duration of taper are listed in Box 3. Routine tapering is probably unnecessary when antidepressants have been prescribed for less than 4 weeks, as discontinuation symptoms are unlikely to occur with such a short duration of treatment. Abruptly stopping an antidepressant is justified if a patient has developed serious sideeffects believed to be due to the antidepressant, there is a medical emergency warranting stopping the

\section{Box 3 Factors that may influence the duration} of taper

- The propensity of the antidepressant to cause discontinuation symptoms

- The dose of the antidepressant

- Whether the patient has a previous history of discontinuation symptoms

- The degree of urgency associated with stopping the antidepressant antidepressant or the antidepressant has induced mania. Further details on the practicalities of tapering antidepressants are provided in the final section of this article, entitled 'Management'.

\section{Tapering and antidepressant switching}

The Tint et al (2007) data imply that if tapering SSRIs and venlafaxine is beneficial in reducing discontinuation symptoms, then it needs to continue for more than 14 days for most patients. When switching antidepressants because of lack of efficacy, a taper in excess of 14 days before starting the new antidepressant is likely to be impractical as it would cause excessive delay before starting the new drug. An abrupt switch or start-taper switch allow the new antidepressant to be started immediately and are preferable, assuming that there are no potential drug interactions that warrant a drug washout period. A start-taper switch refers to starting the new antidepressant and simultaneously gradually tapering the previous one. Whether an abrupt switch or start-taper switch is chosen partly depends on the likelihood of discontinuation symptoms occurring, which in turn depends on the pharmacological similarity between the two antidepressants.

Several case reports describe antidepressant discontinuation symptoms rapidly resolving, usually within $24 \mathrm{~h}$, after a new but pharmacologically similar antidepressant is commenced (e.g. Keuthen et al, 1994; Giakas \& Davis, 1997; Benazzi, 1999) although this is not always the case (Phillips, 1995). This 'cross-suppression' means that an abrupt switch can be used when switching between pharmacodynamically similar agents, for example when switching from one SSRI to another SSRI or an SNRI or when switching between SNRIs. If the pharmacology of the two antidepressants is sufficiently different to suggest that the second agent will not suppress symptoms from discontinuation of the first, then a start-taper switch can be used. An abrupt switch may also be considered when switching between pharmacologically different antidepressants, provided that the patient does not have a history of severe discontinuation symptoms and that both patient and clinician are aware that discontinuation symptoms may arise. Most discontinuation symptoms are mild and many of the associated problems arise when they are unexpected, leading patients to worry about their meaning, or increasing the likelihood of doctors making an incorrect diagnosis.

The Sequenced Treatment Alternatives to Relieve Depression $\left(\mathrm{STAR}^{*} \mathrm{D}\right)$ study used abrupt switching from citalopram (after a mean duration of treatment of 8 weeks) to one of three alternatives: sertraline, venlafaxine and bupropion (Rush et al, 2006). The 
design makes it difficult to comment specifically on discontinuation symptoms after stopping citalopram but the overall side-effect burden did not differ between the three new drugs, despite the lack of similar pharmacology with bupropion. This suggests that abrupt switching is reasonable in this situation. The prospective study reported by Tint et al (2007) also suggests that discontinuation symptoms are not likely to be a major problem with abrupt switching. In this study, mean scores for discontinuation and depressive symptoms increased significantly following stoppage of either an SSRI or venlafaxine and a 1 week antidepressant-free period. Patients then started a new antidepressant chosen on clinical grounds by the treating clinician. One week after starting the new antidepressant, mean scores on both symptom scales had fallen to near baseline values even though about one-third of the patients had been switched to a noradrenergic antidepressant.

It is important to emphasise that a washout period is essential when switching to and from MAOIs because of the risk of drug interactions that can lead to serotonin syndrome. The BNF (British Medical Association \& Royal Pharmaceutical Society of Great Britain, 2007) suggests appropriate washout periods, the durations of which differ depending on the details of the MAOI switch. A washout should also be considered when switching from fluoxetine to a TCA, as the long-half life of fluoxetine, plus its ability to inhibit cytochrome P450 enzymes, could result in elevation of plasma TCA levels, leading to adverse effects.

\section{Choice of antidepressant}

When an antidepressant is selected for a patient the decision should reflect various factors, including the patient's experience of previous antidepressants in terms of efficacy and tolerability, the potential for drug interactions and any cautions or contraindications to prescribing. If a patient has previously experienced a severe discontinuation syndrome or is known to adhere poorly to medication regimens then these factors should also be considered. In such cases an antidepressant with a low propensity to cause discontinuation symptoms (e.g. fluoxetine) may be an appropriate choice.

\section{Education for health professionals}

Several surveys in the late 1990s indicated that many health professionals were unfamiliar with the concept of antidepressant discontinuation symptoms (Young \& Currie, 1997; Donoghue \& Haddad, 1999; Haddad et al, 1999). For example 30\% of UK general practitioners questioned in 1998 (Haddad et al, 1999) reported being poorly aware of antidepressant discontinuation symptoms and, from other questions, it was apparent that many of the remainder had overrated their knowledge. Increased awareness is necessary if clinicians are to adopt preventive strategies and to effectively diagnose and treat discontinuation symptoms when they arise.

\section{Information for patients}

Patients taking antidepressants, or considering antidepressant treatment, need to be provided with information. They need to know that antidepressants should not be stopped abruptly or interrupted as this can cause discontinuation symptoms, and that antidepressants should be tapered at the end of treatment to minimise this risk. It should be explained that although antidepressants can cause discontinuation or withdrawal symptoms they do not cause craving, tolerance and loss of control over medication taking and for this reason antidepressants are not regarded as addictive in the way that alcohol and many illicit drugs are. Providing this information lessens the likelihood of discontinuation symptoms appearing and their misdiagnosis. Although discontinuation symptoms are unlikely to occur when antidepressants have been taken for less than 4 weeks (Haddad, 1998) the intention when starting treatment is to treat for a longer period than this, assuming of course that the drug is effective and well tolerated. Consequently all patients should be warned about discontinuation symptoms before they start taking an antidepressant and also when discontinuation is being contemplated.

\section{Neonatal symptoms}

Symptoms in newborn infants following maternal SSRI use in late pregnancy have been described in case reports (e.g. Haddad et al, 2005), adverse drug reaction reports (Phelan, 2004) and in prospective studies (e.g. Laine et al, 2003). Similar symptoms have been reported following maternal use of TCAs in late gestation (e.g. Cowe et al, 1982). Symptoms include shivering, tremor, restlessness, increased tonus, respiratory difficulties (ranging from transient tachypnoea to cyanosis), irritability, crying, restless sleep and feeding difficulties. Symptoms are either present at birth or commence within a few hours or days after birth and are usually mild and self-limiting, resolving within 2 weeks. Occasionally symptoms are severe, including seizures and hyperpyrexia, and may warrant admission to a neonatal intensive care unit (Haddad et al, 2005). It is unclear to what extent these symptoms represent a neonatal antidepressant discontinuation syndrome, serotonin toxicity (i.e. a direct toxic effect of the antidepressant) or are unrelated to medication (Haddad et al, 2005). 
Different aetiologies may apply in different cases and in some cases a combination of mechanisms may apply. The possibility of discontinuation symptoms arising in breast-fed infants whose mothers suddenly stop antidepressant treatment has been raised (Kent \& Laidlaw, 1995).

It is recommended that the possibility of neonatal symptoms (as well as other adverse effects of antidepressants in the newborn) should be discussed with pregnant or breast-feeding mothers who are contemplating starting or continuing antidepressant treatment. The possible risks need to be balanced against the benefits of antidepressant treatment and breast-feeding and the risks of an untreated depressive illness. Decisions should be made on an individual patient basis.

\section{Management}

The treatment of discontinuation symptoms depends on (i) whether or not further antidepressant medication is warranted and (ii) the severity of the discontinuation symptoms. If further antidepressant treatment is warranted, irrespective of the occurrence of discontinuation symptoms, then restarting the antidepressant will cause rapid resolution of the symptoms. This scenario usually follows failure to take an antidepressant as prescribed, during either the acute or maintenance phase of an illness. If further antidepressant treatment is not clinically indicated then management depends on the severity of the discontinuation symptoms. Most symptoms are mild and in these cases treatment usually requires only that the patient be reassured about their self-limiting nature. Symptoms can be treated symptomatically if they are of moderate severity. For example insomnia may be treated with a short course of a benzodiazepine. Antimuscarinic agents can help in the treatment of gastrointestinal symptoms following TCA discontinuation (Dilsaver et al, 1983a,b), which is consistent with these symptoms being due to cholinergic rebound. If the discontinuation symptoms are severe then the antidepressant can be reinstated, symptoms will usually resolve within $24 \mathrm{~h}$ and then the antidepressant can be withdrawn more cautiously. Treatment should always include an appropriate explanation of the symptoms to the patient and follow-up to ensure that the symptoms have resolved.

These principles of treatment apply not only to the primary TCA and SSRI discontinuation syndromes, but also to rare discontinuation syndromes, although the evidence is only from case reports. For example mania/hypomania following antidepressant discontinuation has been reported to resolve spontaneously after a short duration without treatment (Mirin $e t$ al, 1981; Bloch et al, 1995) and also to respond to restarting the offending antidepressant (Nelson et al, 1983) or commencing antipsychotic treatment (Mirin et al, 1981). Extrapyramidal discontinuation symptoms associated with venlafaxine (Wolfe, 1997), fluvoxamine (Hirose, 2001), imipramine (Sathananthan \& Gershon, 1973) and desipramine (Dilsaver et al, 1983a) have been reported to resolve when the responsible antidepressant has been restarted.

If, when attempting to withdraw and stop an antidepressant, severe discontinuation symptoms appear, either during or at the end of a taper, one should consider increasing the antidepressant dose back to the lowest dose that prevented their appearance, and then commencing a slower taper. Some individuals require very gradual tapers to prevent discontinuation symptoms reappearing (Koopowitz \& Berk, 1995; Amsden \& Georgian 1996; Louie et al, 1996). Some antidepressants, including paroxetine, are available in liquid formulations that allow very gradual tapers. When managing SSRI and SNRI discontinuation symptoms another strategy is to switch to fluoxetine, the SSRI with the longest half-life. According to anecdotal reports, fluoxetine can suppress discontinuation symptoms associated with other SSRIs (Keuthen et al, 1994; Benazzi, 1999) and venlafaxine (Giakas \& Davis, 1997). If the switch is successful, fluoxetine can usually be stopped after several weeks of treatment without discontinuation symptoms reappearing. The effectiveness of this strategy appears to reflect the long half-life of fluoxetine (1.9 days) and its active metabolite norfluoxetine, which has a half-life of 7-15 days (Haddad, 1998).

\section{Declaration of interest}

P. H. has received reimbursement for lecturing and / or consultancy from the manufacturers of several antidepressants, including GlaxoSmithKline, Lilly, Lundbeck and Wyeth. He has been a principal investigator in a clinical trial of duloxetine sponsored by Lilly. I. M. A. has received reimbursement for lecturing and/or consultancy from the manufacturers of several antidepressants, including Lilly, Lundbeck and Wyeth.

\section{References}

American Psychiatric Association (1994) Diagnostic and Statistical Manual of Mental Disorders (4th edn) (DSM-IV). APA.

Amsden, G. W. \& Georgian, F. (1996) Orthostatic hypotension induced by sertraline withdrawal. Pharmacotherapy, 16, 684-686.

Andersen, H. \& Kristiansen, E. S. (1959) Tofranil treatment of endogenous depressions. Acta Psychiatrica Scandinavica, 34, 387-397.

Anderson, I. M., Nutt, D. J. \& Deakin J. F. (2000) Evidencebased guidelines for treating depressive disorders with 
antidepressants: a revision of the 1993 British Association for Psychopharmacology guidelines. Journal of Psychopharmacology, 14, 3-20.

Baldwin, D. S., Montgomery, S. A., Nil, R., et al (2007) Discontinuation symptoms in depression and anxiety disorders. International Journal of Neuropsychopharmacology, 10, 73-84.

BBC Panorama (2002) The secrets of seroxat. 14 October. http: / / news. bbc.co.uk/1/hi/programmes/panorama/2317249.stm

BBC Panorama (2003) Seroxat: Emails from the edge. 11 May. http:/ / news.bbc.co.uk/1/hi/programmes/panorama/2982797. stm

Benazzi, F. (1996) Venlafaxine withdrawal symptoms. Canadian Journal of Psychiatry, 41, 487.

Benazzi, F. (1999) Selective serotonin reuptake inhibitor discontinuation syndrome: putative mechanisms and prevention strategies. Canadian Journal of Psychiatry, 44, 95-96.

Black, K., Shea, C., Dursun, S., et al (2000) Selective serotonin reuptake inhibitor discontinuation syndrome: proposed diagnostic criteria. Journal of Psychiatry and Neuroscience, 25, 255-261.

Bloch, M., Stager, S. V., Braun, A. R., et al (1995) Severe psychiatric symptoms associated with paroxetine withdrawal. Lancet, $346,57$.

Bogetto, F., Bellino, S., Revello, R. B., et al (2002) Discontinuation syndrome in dysthymic patients treated with selective serotonin reuptake inhibitors: a clinical investigation. CNS Drugs, 16, 273-283.

British Medical Association \& Royal Pharmaceutical Society of Great Britain (2007) British National Formulary (March issue). BMJ Publishing Group \& RPS Publishing.

Committee on Safety on Medicines (2004) Report of the CSM Expert Working Group on the Safety of Selective Serotonin Reuptake Inhibitor Antidepressants. CSM. http://www.mhra.gov. uk/home/groups/pl-p/documents/drugsafetymessage/ con019472.pdf

Cowe, L., Lloyd, D. J. \& Dawling, S. (1982) Neonatal convulsions caused by withdrawal from maternal clomipramine. BMJ (Clinical Research Edition), 19, 1837-1838.

Demyttenaere, K., Mesters, P., Boulanger, B., et al (2001) Adherence to treatment regimen in depressed patients treated with amitriptyline or fluoxetine. Journal of Affective Disorders, 65, 243-252.

Dilsaver, S. C., Kronfol, Z., Greden, J. F., et al (1983a) Antidepressant withdrawal syndromes: evidence supporting the cholinergic overdrive hypothesis. Journal of Clinical Psychopharmacology, 3, 157-164.

Dilsaver, S. C., Feinberg, M. \& Greden, J. F. (1983b) Antidepressant withdrawal symptoms treated with anticholinergic agents. American Journal of Psychiatry, 140, 249-251.

Dominguez, R. A. \& Goodnick, P. J. (1995) Adverse events after the abrupt discontinuation of paroxetine. Pharmacotherapy, 15, 778-780.

Donoghue, J. \& Haddad, P. (1999) Pharmacists lack knowledge of antidepressant discontinuation symptoms. Journal of Clinical Psychiatry, 60, 124-125.

Drug and Therapeutics Bulletin (1999) Withdrawing patients from antidepressants. Drug and Therapeutics Bulletin, 37 (July), 49-52.

Einbinder, E. (1995) Fluoxetine withdrawal? American Journal of Psychiatry, 152, 1253.

Fava, M., Mulroy, R., Alpert, J., et al (1997) Emergence of adverse effects following discontinuation of treatment with extendedrelease venlafaxine. American Journal of Psychiatry, 154, 17601762.

Frost, L. \& Lal, S. (1995) Shock-like sensations after discontinuation of selective serotonin reuptake inhibitors. American Journal of Psychiatry, 152, 810.

Giakas, W. J. \& Davis, S. M. (1997) Intractable withdrawal from venlafaxine treated with fluoxetine. Psychiatric Annals, 27, 85-86.

Goldstein, T. R., Frye, M. A., Denicoff, K. D., et al (1999) Antidepressant discontinuation-related mania: critical prospective observation and theoretical implications in bipolar disorder. Journal of Clinical Psychiatry, 60, 563-67
Haddad, P. (1998) The SSRI discontinuation syndrome. Journal of Psychopharmacology, 12, 305-313.

Haddad, P. (1999) Do antidepressants have any potential to cause addiction? Journal of Pychopharmacology, 13, 300-307.

Haddad, P. M. (2005) Do antidepressants cause dependence? Epidemiologia e Psichiatria Sociale, 14(2), 58-62.

Haddad, P. \& Anderson, I. (1999) Antidepressants aren't addictive: clinicians have depended on them for years. Journal of Psychopharmacology, 13, 291-292.

Haddad, P. M. \& Qureshi, M. (2000) Misdiagnosis of antidepressant discontinuation symptoms. Acta Psychiatrica Scandinavica, 102, 466-468.

Haddad, P., Tylee, A. \& Young, A. P. (1999) General practitioners' knowledge of antidepressant discontinuation reactions (abstract). Journal of Psychopharmacology, 13 (suppl. A), A44.

Haddad, P. M., Devarajan, S. \& Dursun, S. M. (2001) Antidepressant discontinuation symptoms presenting as 'stroke'. Journal of Psychopharmacology, 15, 139-141.

Haddad, P. M., Pal, B. R., Clarke, P., et al (2005) Neonatal symptoms following maternal paroxetine treatment: serotonin toxicity or paroxetine discontinuation syndrome? Journal of Psychopharmacology, 19, 554-557.

Halle, M. T. \& Dilsaver, S. C. (1993) Tranylcypromine withdrawal phenomena. Journal of Psychiatry and Neuroscience, 18, 49-50.

Hirose, S. (2001) Restlessness related to SSRI withdrawal. Psychiatry and Clinical Neurosciences, 55, 79-80.

Judge, R., Parry, M. G., Quail, D., et al (2002) Discontinuation symptoms: comparison of brief interruption in fluoxetine and paroxetine treatment. International Clinical Psychopharmacology, 17, 217-225.

Kent, L. S. W. \& Laidlaw, J. D. D. (1995) Suspected congenital sertraline dependence. British Journal of Psychiatry, 167, 412-413.

Keuthen, N. J., Cyr, P., Ricciardi, J. A., et al (1994) Medication withdrawal symptoms in obsessive-compulsive disorder patients treated with paroxetine. Journal of Clinical Psychopharmacology, 14, 206-207.

Koopowitz, L. F. \& Berk, M. (1995) Paroxetine induced withdrawal effects. Human Psychopharmacology, 10, 147-148.

Kramer, J. C., Klein, D. F. \& Fink, M. (1961) Withdrawal symptoms following discontinuation of imipramine therapy. American Journal of Psychiatry, 118, 549-550.

Laine, K., Heikkinen, T., Ekblad, U., et al (2003) Effects of exposure to selective serotonin reuptake inhibitors during pregnancy on serotonergic symptoms in newborns and cord blood monoamine and prolactin concentrations. Archives of General Psychiatry, 60, 720-726.

Liskin, B., Roose, S. \& Walsh, T. (1984) Acute psychosis following phenelzine discontinuation. Journal of Clinical Psychopharmacology, 5, 46-47.

Louie, A. K., Lannon, R. A., Kirsch, M. A., et al (1996) Venlafaxine withdrawal reactions. American Journal of Psychiatry, 153, 1652.

MacCall, C. \& Callender, J. (1999) Mirtazapine withdrawal causing hypomania. British Journal of Psychiatry, 175, 390.

Mann, A. M. \& MacPherson, A. (1959) Clinical experience with imipramine (G22355) in the treatment of depression. Canadian Psychiatric Association Journal, 4, 38-47.

Medawar, C. (1997) The antidepressant web - marketing depression and making medicines work. International Journal of Risk and Safety in Medicine, 10, 75-126.

Michelson, D., Fava, M., Amsterdam, J., et al (2000) Interruption of selective serotonin reuptake inhibitor treatment. Doubleblind, placebo-controlled trial. British Journal of Psychiatry, 176, 363-368.

Mirin, S. M., Schatzberg, A. F. \& Creasey, D. E. (1981) Hypomania and mania after withdrawal of tricyclic antidepressants. American Journal of Psychiatry, 138, 87-89.

Nelson, J. C., Schottenfeld, R. S. \& Conrad, E. D. (1983) Hypomania after desipramine withdrawal. American Journal of Psychiatry, 140, 624-625.

Pacheco, L., Malo. P., Aragues, E., et al (1996) More cases of paroxetine withdrawal syndrome. British Journal of Psychiatry, $169,384$.

Perahia, D. G., Kajdasz, D. K., Desaiah, D., et al (2005) Symptoms following abrupt discontinuation of duloxetine treatment in 
patients with major depressive disorder. Journal of Affective Disorders, 89, 207-212.

Phelan, P. (2004) Neonatal Withdrawal Syndrome with Serotonin Reuptake Inhibitors. Office of Drug Safety Review. Food and Drug Administration. http:/ / www.fda.gov/ohrms/dockets / ac/04/slides/2004-4050S1_10_Phelan.ppt

Phillips, S. D, (1995) A possible paroxetine withdrawal syndrome. American Journal of Psychiatry, 152, 645-646.

Price, J. S., Waller, P. C., Wood, S. M., et al (1996) A comparison of the post-marketing safety of four selective serotonin re-uptake inhibitors, including the investigation of symptoms occurring on withdrawal. British Journal of Clinical Pharmacology, 42 757-763.

Rosenbaum, J. F., Fava, M., Hoog, S. L., et al (1998) Selective serotonin reuptake inhibitor discontinuation syndrome: a randomised clinical trial. Biological Psychiatry, 44, 77-87.

Roth, S. D. (1985) More on psychosis following phenelzine discontinuation. Journal of Clinical Psychopharmacology, 5, 360-361.

Rothschild, A. J. (1985) Mania after withdrawal of isocarboxazid. Journal of Clinical Psychopharmacology, 5, 340-342.

Rush, A. J., Trivedi, M. H., Wisniewski, S. R., et al (2006) Bupropion-SR, sertraline, or venlafaxine-XR after failure of SSRIs for depression. New England Journal of Medicine, 354 1231-1242.

Sathananthan, G. L. \& Gershon, S. (1973) Imipramine withdrawal: an akathisia-like syndrome. American Journal of Psychiatry, 130 1286-87

Schatzberg, A. F., Haddad, P., Kaplan, E. M., et al (1997) Possible biological mechanisms of the serotonin reuptake inhibitor discontinuation syndrome. Journal of Clinical Psychiatry, 58 (suppl. 7), 23-27.

Schatzberg, A. F., Blier, P., Delgado, P. L., et al (2006) Antidepressant discontinuation syndrome: consensus panel recommendations for clinical management and additional research. Journal of Clinical Psychiatry, 67 (suppl 4), 27-30.

Stoukides, J. A. \& Stoukides, C. A. (1991) Extrapyramidal symptoms upon discontinuation of fluoxetine. American Journal of Psychiatry, 148, 1263.

Szabadi, E. (1992) Fluvoxamine withdrawal syndrome. British Journal of Psychiatry, 160, 283-284.

Tint, A., Haddad, P. M. \& Anderson, I. M. (2007) The effect of rate of antidepressant tapering on the incidence of discontinuation symptoms: a randomised study. Journal of Psychopharmacology, in press.

Tyrer, P. (1984) Clinical effects of abrupt withdrawal from tricyclic antidepressants and monoamine oxidase inhibitors after long term treatment. Journal of Affective Disorders, 6, 1-7.

Tyrer, P. (1999) Stress diathesis and pharmacological dependence. Journal of Psychopharmacology, 13, 294-295.

Vandel, P., Sechter, D., Weiller, E., et al (2004) Post-treatment emergent adverse events in depressed patients following treatment with milnacipran and paroxetine. Human Psychopharmacology, 19, 585-586.

Wolfe, R. M. (1997) Antidepressant withdrawal reactions. American Family Physician, 56, 452-462.

World Health Organization (1992) The ICD-10 Classification of Mental and Behavioural Disorders. World Health Organization.

Young, A. H. \& Currie, A. (1997) Physicians' knowledge of antidepressant withdrawal effects: a survey. Journal of Clinical Psychiatry, 58 (suppl. 7), 28-30.

\section{MCQs}

1 Discontinuation symptoms are recognised with:

a only tricyclic antidepressants

b only monoamine oxidase inhibitors

c only SSRIs

d only SNRIs

e all major antidepressant classes.
2 Characteristic features of antidepressant discontinuation symptoms include:

a onset of symptoms about 2 weeks after the antidepressant is stopped

b usually severe symptoms

c symptom duration of 4 weeks or longer if untreated

$\mathrm{d}$ resolution of symptoms within $24 \mathrm{~h}$ of restarting the antidepressant

e symptoms that are more marked in men than in women.

3 Key principles in preventing and managing antidepressant discontinuation symptoms include:

a warning patients about the addictive nature of antidepressants

b tapering antidepressants at the end of treatment

c advocating drug holidays to avoid sexual sideeffects

d restarting antidepressant treatment whenever discontinuation symptoms are suspected

e avoiding direct switching from one SSRI to another SSRI.

4 Characteristic symptoms of SSRI discontinuation reactions are:

a auditory hallucinations

b emotional numbing

c electric-shock-like sensations

d obsessional thoughts

e disorientation.

5 SSRI discontinuation symptoms are:

a seen only in adults

b more common with fluoxetine than with other SSRIs

c more common with a shorter, as opposed to a longer, duration of antidepressant treatment

d seen only when SSRIs are used in the treatment of depressive disorders

e more likely with higher antidepressant doses.

\section{MCO answers}

$\begin{array}{llllllll}1 & & 2 & & 3 & & 4 & 5 \\ \text { a F } & \text { a F } & \text { a F } & \text { a F } & \text { a F } \\ \text { b F } & \text { b F } & \text { b T } & \text { b F } & \text { b F } \\ \text { c F } & \text { c F } & \text { c F } & \text { c T } & \text { c F } \\ \text { d F } & \text { d T } & \text { d F } & \text { d F } & \text { d F } \\ \text { e T } & \text { e F } & \text { e F } & \text { e F } & \text { e T }\end{array}$

